\title{
Adesão à técnica de lavagem de mãos em Unidade de Terapia Intensiva Neonatal
}

\author{
Compliance with hand washing technique in a Neonatal Intensive Care Unit
}

\author{
Mariana Reclusa Martinez ${ }^{1}$, Luiz Alexandre A. F. Campos ${ }^{1}$, Paulo Cesar K. Nogueira ${ }^{3}$
}

\section{RESUMO}

Objetivo: Avaliar o cumprimento da técnica de lavagem das mãos empregadas em uma unidade de terapia intensiva neonatal (UTIN) pelos profissionais de saúde e visitantes.

Métodos: Estudo prospectivo e observacional em uma UTIN universitária em Santos (SP). Observações foram feitas nos períodos da manhã e da tarde por um período de sete meses. Os dados foram coletados por dois estudantes de medicina em planilha específica. As pessoas observadas não sabiam da natureza do trabalho. Para comparar os resultados obsevados entre categorias profissionais e entre períodos de observação, utilizou-se o teste do qui-quadrado ou exato de Fisher, com duas abordagens: a) análise por intenção de tratar (considerando o número total de indivíduos e assumindo que aqueles que não lavaram as mãos adotaram o procedimento incorreto) e b) análise por protocolo (apenas aqueles que lavaram as mãos foram considerados). Rejeitou-se a hipótese de nulidade se $p<0,05$.

Resultados: Foram realizadas 43 observações com duração de cerca de 30 minutos cada uma, sendo 20 minutos durante a manhã e 23 à tarde. Entre as pessoas analisadas, seis (14\%) eram médicos, $26(60 \%)$ da equipe de enfermagem, três (7\%) técnicos de laboratório e raio X e oito (19\%) acompanhantes de pacientes. Dentre os observados, 24 (56\%) lavaram as mãos antes de entrar na unidade, sendo a lavagem observada com maior frequência no período da manhã (75\%) do que à tarde (39\%). A técnica correta não foi observada em nenhuma das vezes.

Conclusões: A técnica de lavagem das mãos raramente é adequada e, por isso, programas educacionais para aumentar a adesão dos profissionais de saúde são importantes.
Palavras-chave: Unidade de Terapia Intensiva; recémnascido; infecção hospitalar; lavagem de mãos; pessoal de saúde.

\section{ABSTRACT}

Objective: To evaluate the hand washing technique employed by health professionals and visitors of patients treated in a Neonatal Intensive Care Unit (NICU).

Methods: A prospective observational study was performed in the NICU of a teaching hospital in Santos, São Paulo, Brazil. The evaluation was made during seven months, with mornings and afternoons observations. Data were collected by two medical students. Observed people were not informed of the objective of the research. Two approaches were used to compare the hand washing technique: a) analysis by intention to treat, considering individuals who did not wash their hands as using an inadequate technique and b) by protocol analysis, considering only those who washed their hands. The chi-square or Fisher's exact tests were used to compare the groups and $p<0.05$ was adopted in all tests to reject the null hypothesis.

Results: 43 observations were performed, which lasted about 30 minutes each, being 20 in the morning and 23 in the afternoon. We observed six physicians (14\%), 26 nurses $(60 \%)$, three (7\%) laboratory and X-ray technicians and eight (19\%) relatives of patients. Among these, $24(55.8 \%)$ washed their hands before entering the unit. The procedure was adopted more frequently during the morning (75\%) than in the afternoon (39\%). The correct technique was never adopted by any observed category.

Endereço para correspondência:

Mariana Reclusa Martinez

Rua Doutor Francisco de Souza Dantas, 45/31 - Marapé

CEP 11070-160 - Santos/SP

E-mail: marireclusa@gmail.com

Fonte financiadora: Programa de Iniciação Científica do Centro Universitário Lusíada

Recebido em: 25/7/08

Aceito em: 17/12/08 
Conclusions: Hand washing techniques are rarely followed in hospitals and, therefore, educational programs to increase the compliance with health professionals are urgent.

Key-words: Intensive Care Unit; newborn infant; cross infection; handwashing; health personnel.

\section{Introdução}

A infecção relacionada à assistência hospitalar é um sério problema de Saúde Pública que afeta um número grande de pacientes, aumentando o tempo de internação, o risco de mortalidade e os custos socioeconômicos ${ }^{(1-4)}$. Nos EUA, ocorrem cerca de 2 milhões de casos anuais de infecções hospitalares, levando a 20 mil óbitos e gerando um gasto estimado em 4,5 a 5,7 bilhões de dólares por ano ${ }^{(5)}$. Uma pesquisa realizada em 103 hospitais terciários de 13 capitais brasileiras mostrou que a taxa de infecção foi de $13 \%$, sendo que a prevalência na Unidade de Terapia Intensiva neonatal (UTIN) foi a mais alta $(46,9 \%)$, seguida pela Unidade de Queimados $(38,2 \%)$ e pela UTI pediátrica $(32,9 \%)^{(6)}$.

De acordo com o Centers for Disease Control (CDC), são consideradas infecções neonatais hospitalares aquelas adquiridas no período intraparto (de origem materna e manifestada 48 horas de vida), durante a hospitalização ou até 48 horas após a alta, com exceção às infecções transplacentárias ${ }^{(3)}$. Nos neonatos, o risco de infecções é mais elevado devido à imaturidade do seu sistema imunológico, à necessidade de procedimentos invasivos inerentes ao suporte vital, com a presença de cânula traqueal e cateteres centrais, entre outros, além do uso de medicações que aumentam o risco de infecção (por exemplo, bloqueadores $\mathrm{H} 2$ e corticosteroides) $)^{(3,7)}$.

Cerca de 30\% dos casos de infecções relacionadas à assistência à saúde são considerados preveníveis por medidas simples, sendo a lavagem correta das mãos pelos profissionais de saúde a mais efetiva delas. São as mãos que transportam o maior número de micro-organismos aos pacientes, por meio contato direto ou através de objetos ${ }^{(1,5,7-10)}$. Dentre esses micro-organismos, muitos são patógenos potenciais, como $S$. aureus, E. coli, $P$. aeruginosa e E. fecaelis, sendo metade deles multirresistente aos antibióticos ${ }^{(1)}$.

A lavagem das mãos é indicada antes de ministrar medicamentos por via oral e preparar a nebulização, antes e após a realização de trabalhos hospitalares, atos e funções fisiológicas ou pessoais, antes e depois do manuseio de cada paciente, do preparo de materiais ou equipamentos, da coleta de espécimes, da aplicação de medicamentos injetáveis e da higienização e troca de roupa dos pacientes ${ }^{(11)}$. Apesar de a importância da transmissão de infecções relacionadas à assistência à saúde pelo contato das mãos ser aceita mundialmente, o cumprimento das normas técnicas para a sua prevenção é limitado, principalmente entre os profissionais da categoria médica, tanto em países desenvolvidos quanto em países em desenvolvimento, sendo inferior a $50 \%{ }^{(1,8,9)}$.

Nesse contexto, o presente estudo tem por objetivo avaliar o cumprimento das técnicas de lavagem das mãos empregadas em uma UTIN pelos profissionais de saúde e familiares visitantes.

\section{Métodos}

Realizou-se um estudo prospectivo e observacional na UTIN de um hospital de ensino público estadual de atendimento terciário na cidade de Santos (SP). A referida unidade neonatal é composta por 13 leitos, distribuidos em quatro quartos abertos para uma área central comum e é disponibilizada apenas para nascidos na maternidade desse hospital. Há uma pia antes da entrada e dentro de cada quarto. As torneiras são manuais, sem sensores, e o sabão disponível para a lavagem das mãos é clorexidina a $4 \%$, em solução degermante.

Foram avaliados diversos profissionais da área da saúde, entre eles, médicos, enfermeiros, técnicos e auxiliares de enfermagem, fonoaudiólogos, fisioterapeutas, técnicos de laboratório e de raio X, estudantes de medicina, professores, residentes e os familiares dos pacientes internados. As observações foram feitas por dois estudantes de medicina que estiveram na UTIN nos períodos da manhã e da tarde por cerca de uma hora durante sete meses, entre maio de 2007 e janeiro de 2008, totalizando 23 horas de observação. Os indivíduos sob observação não estavam cientes dos objetivos ou da natureza do estudo.

Anotou-se a categoria profissional (profissional de saúde ou familiar do paciente internado), o período do dia (manhã ou tarde), o cumprimento da técnica correta (segundo o manual do Ministério da Saúde) $)^{(11)}$, que consistia em retirar joias, pulseiras e relógios, ter unhas curtas, lavar palma, dorso, espaços interdigitais, extremidades dos dedos e unhas, polegar, punhos, enxaguar satisfatoriamente (retirando totalmente a espuma e os resíduos de sabão) e fechar a torneira com papel toalha. O tipo de manipulação realizada no paciente 
também foi anotado e categorizado em procedimento não invasivo ou invasivo.

Os procedimentos não invasivos observados quanto à lavagem das mãos foram: antes e após o preparo de medicamentos, antes e após a punção venosa, antes e após a troca de fraldas, antes da realização de raio $\mathrm{X}$ e nos intervalos dos exames radiológicos entre uma criança e outra. Os procedimentos invasivos analisados foram: cateterismo vesical, aspiração orotraqueal, cateterização de veia central ou periférica e sondagens oro- ou nasogástricas.

O tempo gasto durante a lavagem das mãos foi divido em: menos de dez segundos, de 11 a 20 segundos, de 21 a 30 segundos e mais de 30 segundos. Considerou-se ideal o tempo de pelo menos dez segundos. O hospital disponibiliza detergente líquido (clorhexedine 4\%) para o procedimento de higienização das mãos e não se observou a presença de álcool em gel em nenhum local da unidade.

Os dados foram coletados em planilha específica, adaptada a partir de um instrumento previamente testado e validado, contendo as etapas das lavagens das mãos, o tempo gasto e as situações em que se lavaram as mãos ${ }^{(7)}$. Para que não houvesse viés, os profissionais só foram informados da pesquisa ao final da coleta dos dados, quando se solicitou o consentimento esclarecido dos participantes. Durante toda a pesquisa, tomou-se o cuidado de não revelar a identidade pessoal dos indivíduos observados, uma vez que o objetivo da pesquisa não era avaliar o comportamento de indivíduos em particular e sim o cumprimento de normas técnicas por todos os freqüentadores da unidade. De qualquer forma, tomou-se o cuidado de apenas avaliar e divulgar os resultados após a assinatura dos termos de consentimento livre e esclarecido.

Para a análise dos dados, foi feita a estatística descritiva dos resultados na forma de tabelas de frequência, uma vez que todas as variáveis são qualitativas. Para comparação dos grupos, utilizou-se o teste do qui-quadrado ou exato de Fisher. Na comparação das técnicas de lavagem das mãos, utilizaram-se duas abordagens: a) análise por intenção de tratar, na qual se considerou o número total de indivíduos observados, assumindo que aqueles que não lavaram as mãos adotaram procedimento inadequado e b) análise por protocolo, na qual apenas os indivíduos que lavaram as mãos foram considerados quanto à correção das técnicas. Para todos os testes, adotou-se $p<0,05$ para rejeitar a hipótese de nulidade. A amostra analisada foi de conveniência, buscando-se representar o que ocorre na unidade durante o período do dia (manhã e tarde). As observações foram estendidas até o momento em que os indivíduos já observados começaram a repetir o procedimento, ou seja, evitou-se que as mesmas pessoas fossem observadas mais de uma vez.

\section{Resultados}

Foram realizadas 43 observações, sendo 20 delas durante a manhã (47\%) e 23 à tarde (53\%). Entre as pessoas analisadas, seis eram médicos (14\%), 26 da equipe de enfermagem, entre eles, enfermeiros e auxiliares de enfermagem (60\%), três (7\%) técnicos de laboratório e raio $\mathrm{X}$ e oito (19\%) acompanhantes de pacientes, que correspondiam às mães dos recém-nascidos. Embora não tenha sido feita a pesquisa dos dados demográficos dos indivíduos observados, a característica predominante dessa amostra é ser constituída por profissionais de saúde jovens, com alguns anos de vivência na unidade, não havendo, durante o período de estudo, indivíduos recém-admitidos para o trabalho na unidade.

Ao entrar na unidade, somente 24 das 43 pessoas observadas lavaram as mãos (56\%). Porém, mais seis pessoas lavaram as mãos em algum momento posterior à entrada na unidade. $\mathrm{Na}$ análise da técnica correta de lavagem, essas seis pessoas foram incluídas, totalizando trinta pessoas que lavaram as mãos (70\%).

A Tabela 1 mostra os resultados obtidos na lavagem das mãos de acordo com a categoria profissional, chamando-se atenção para o fato de que os médicos foram a categoria que menos lavou as mãos, embora a diferença não tenha sido estatisticamente significante.

Os resultados obtidos na comparação da técnica da lavagem das mãos entre todos os indivíduos observados, rotulan-

Tabela 1 - Lavagem das mãos por categoria

\begin{tabular}{lcccc}
\hline & $\begin{array}{c}\text { Médicos } \\
(\mathbf{n = 6 )}\end{array}$ & $\begin{array}{c}\text { Enfermagem } \\
(\mathbf{n = 2 6 )}\end{array}$ & $\begin{array}{c}\text { Outros } \\
(\mathbf{n = 1 1 )}\end{array}$ & $\boldsymbol{p}$ \\
\hline $\operatorname{Sim}$ & $3(50 \%)$ & $19(73 \%)$ & $8(73 \%)$ & $>0,05$ \\
$\operatorname{Não}$ & $3(50 \%)$ & $7(27 \%)$ & $3(27 \%)$ & \\
\hline
\end{tabular}


do-se a ação daqueles que não lavaram as mãos como técnica inadequada (análise por intenção de tratar), estão expressos na Tabela 2, sendo notável que os piores resultados dizem respeito à lavagem de unhas e extremidades dos dedos, com destaque para o fato de que nenhum dos 11 indivíduos da categoria "outros" (familiares e funcionários, com exceção aos médicos e enfermeiros) tenha feito a lavagem da região.

Já os dados obtidos ao se analisar apenas aqueles que lavaram as mãos, excluindo-se os indivíduos que não o fizeram (análise por protocolo), estão expressos na Tabela 3 que, de modo geral, mostrou técnica mais apurada dos profissionais da unidade (médicos e enfermeiros) em comparação aos outros. No entanto, é necessário enfatizar que a técnica correta da lavagem de mãos não foi observada em nenhuma categoria profissional analisada, pois ninguém fechou a torneira com papel toalha.

Quanto à frequência de lavagem das mãos por período, observa-se que a frequência de lavagem das mãos ao entrar na UTIN foi maior no período da manhã $(15 / 20,75 \%) \mathrm{em}$ comparação ao período da tarde $(9 / 23,39 \%)$, diferença essa significativa $(p=0,02)$.

No que diz respeito ao tempo gasto durante a lavagem, 13 pessoas gastaram menos de dez segundos (43\%), 12 gastaram entre dez e 20 segundos (40\%), quatro gastaram entre 20 e 30 segundos (13\%) e apenas uma pessoa gastou mais de 30 segundos (3\%).

Tendo em vista os 22 procedimentos não invasivos, em $44 \%(n=9)$ das vezes as mãos não foram lavadas antes do preparo de medicamentos e, em $100 \%$ da amostra, as mãos não foram lavadas após o preparo das medicações. Já na punção venosa, os profissionais lavaram as mãos antes e após a realização do procedimento em $67 \%$ das 6 observações. No que diz respeito à troca de fraldas $(n=5)$, a lavagem das mãos foi observada em $100 \%$ das vezes antes da realização do procedimento e, em $80 \%$, depois. Antes e após realização de raio $\mathrm{X}$ das crianças, as mãos foram lavadas em $100 \%$ dos casos.

Quanto aos procedimentos invasivos, as mãos foram lavadas $50 \%$ das vezes antes da aspiração orotraqueal $(n=2)$ e nenhuma vez após a realização da aspiração. Já nas sondagens oro e nasogástricas, as mãos foram lavadas em $100 \%$ das três observações tanto antes quanto após a realização do procedimento.

\section{Discussão}

A higiene das mãos é a principal medida para se reduzirem infecções intra-hospitalares e, embora seja um procedimento

Tabela 2 - Procedimentos realizados para lavagem das mãos, considerando-se a não lavagem das mãos como não realização de nenhum dos procedimentos

\begin{tabular}{llcccc}
\hline & $\begin{array}{c}\text { Médico } \\
\mathbf{n}(\%)\end{array}$ & $\begin{array}{c}\text { Enfermagem } \\
\mathbf{n}(\%)\end{array}$ & $\begin{array}{c}\text { Outros } \\
\mathbf{n}(\%)\end{array}$ & Total & $\boldsymbol{p}$ \\
\hline Lavagem das mãos ao entrar & $2(33)$ & $15(58)$ & $7(64)$ & 24 & NS \\
Retirou joias, pulseiras, relógios & $3(50)$ & $12(46)$ & $4(36)$ & 19 & NS \\
Possui unhas curtas & $3(50)$ & $19(73)$ & $5(45)$ & 27 & NS \\
Técnica da lavagem & & & & & NS \\
Palma a palma & $3(50)$ & $19(73)$ & $8(73)$ & 30 & NS \\
Palma com dorso & $3(50)$ & $17(65)$ & $5(45)$ & 25 & NS \\
Espaços interdigitais & $3(50)$ & $8(31)$ & $1(9)$ & 12 & NS \\
Polegar & $3(50)$ & $4(15)$ & $1(9)$ & 8 & 0,03 \\
Unhas e extremidades dos dedos & $3(50)$ & $4(15)$ & 0 & 7 & NS \\
Punhos & $3(50)$ & $4(15)$ & $1(9)$ & 8 & NS \\
Fechou torneira com papel toalha & 0 & 0 & 0 & 18 & NS \\
Enxague satisfatório* & $3(50)$ & $9(37)$ & $6(55)$ & & NS \\
Duração da lavagem & & & $5(45)$ & 13 & NS \\
0 a 10 segundos & $2(33)$ & $6(23)$ & $1(9)$ & 12 & NS \\
10 a 20 segundos & $1(17)$ & $10(38)$ & $2(18)$ & 4 & NS \\
20 a 30 segundos & 0 & $2(8)$ & 0 & 1 & \\
Mais de 30 segundos & 0 & $1(4)$ & &
\end{tabular}

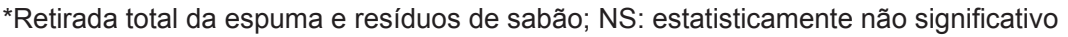


Tabela 3 - Procedimentos realizados na lavagem das mãos, considerando-se apenas aqueles que realmente lavaram a mão

\begin{tabular}{|c|c|c|c|c|c|}
\hline & $\begin{array}{l}\text { Médico } \\
\text { n (\%) }\end{array}$ & $\begin{array}{l}\text { Enfermagem } \\
\mathrm{n}(\%)\end{array}$ & $\begin{array}{l}\text { Outros } \\
n(\%)\end{array}$ & Total & $p$ \\
\hline Lavagem das mãos ao entrar & $2(67)$ & $15(79)$ & $7(87)$ & 24 & NS \\
\hline Retirou joias, pulseiras, relógios & $3(100)$ & $12(63)$ & $4(50)$ & 19 & NS \\
\hline $\begin{array}{l}\text { Possui unhas curtas } \\
\text { Técnica da lavagem }\end{array}$ & $3(100)$ & $19(100)$ & $5(62)$ & 27 & 0,01 \\
\hline Palma a palma & $3(100)$ & $19(100)$ & $8(100)$ & 30 & NS \\
\hline Palma com dorso & $3(100)$ & $17(89)$ & $5(62)$ & 25 & NS \\
\hline Espaços interdigitais & $3(100)$ & $8(42)$ & $1(12)$ & 12 & 0,029 \\
\hline Polegar & $3(100)$ & $4(21)$ & $1(12)$ & 8 & 0,009 \\
\hline Unhas e extremidades dos dedos & $3(100)$ & $4(21)$ & 0 & 7 & 0,002 \\
\hline Punhos & $3(100)$ & $4(21)$ & $1(12)$ & 8 & 0,009 \\
\hline Fechou a torneira com papel toalha & 0 & 0 & 0 & 0 & NS \\
\hline Enxague satisfatório* & $3(100)$ & $9(47)$ & $6(75)$ & 18 & NS \\
\hline \multicolumn{6}{|l|}{ Duração da lavagem } \\
\hline 0 a 10 segundos & $2(67)$ & $6(32)$ & $5(62)$ & 13 & NS \\
\hline 10 a 20 segundos & $1(33)$ & $10(53)$ & $1(12)$ & 12 & NS \\
\hline 20 a 30 segundos & 0 & $2(11)$ & $2(25)$ & 4 & NS \\
\hline Mais de 30 segundos & 0 & $1(5)$ & 0 & 1 & NS \\
\hline
\end{tabular}

*Retirada total da espuma e resíduos de sabão; NS: estatisticamente não significativo

simples e barato, a falta de adesão dos profissionais de saúde é um problema em todo o mundo. Nosso principal resultado revela que, em hospital de ensino de atendimento terciário, apenas $56 \%$ das pessoas analisadas lavaram as mãos ao entrar no serviço de terapia intensiva neonatal, o que não difere da literatura mundial ${ }^{(15-17)}$.

Estudos como o de Lam ${ }^{(14)}$, realizado na UTIN do Hospital Queen Mary da Universidade de Hong Kong, mostraram taxa de adesão à lavagem de mãos em torno de 40\% dentre 666 procedimentos que necessitavam lavagem de mãos antes de contatos com pacientes observados. Já, no trabalho de Won ${ }^{(12)}$, realizado na UTIN do Hospital da Universidade Nacional de Taiwan, observou-se, entre médicos e profissionais de enfermagem, apenas $43 \%$ de adesão à técnica nos episódios para os quais havia necessidade de lavagem de mãos: a) antes e após o contato com pacientes, b) após contato com uma fonte potencial de micro-organismos, como secreções, membranas mucosas, solução de continuidade cutânea, objetos inanimados potencialmente contaminados e c) após retirada de luvas. Já no estudo de Pittet ${ }^{(8)}$, realizado no Hospital da Universidade de Genebra e observando-se exatamente as mesmas situações vistas no trabalho de Won $^{(12)}$, demonstrou-se que em 2.834 oportunidades para lavagem de mãos de 1.043 funcionários do hospital (520 enfermeiras, 158 médicos e 365 outros profissionais), houve apenas $48 \%$ de aderência ao protocolo de higienização das mãos. De maneira geral, tais trabalhos têm resultados similares aos dados aqui apresentados, apesar do limitado número de observações.
Como já relatado em outros estudos, a maior parte das procedimentos analisados envolve a equipe de enfermagem, que apresenta maior proximidade com o paciente por tempo prolongado. Anotou-se cerca de $60 \%$ das observações referentes a essa categoria de profissional de saúde, sendo também a categoria que apresentou maior adesão à higiene das mãos (73\%). Resultados similares foram encontrados em outros estudos da literatura, como o de Pittet, realizado na Universidade de Genebra ${ }^{(8)}$, e o de Mendonça, na UTIN de um hospital público de Goiânia ${ }^{(7)}$. A adesão à técnica, no que diz respeito a outros profissionais de saúde, como técnicos de raio $\mathrm{X}$ e de laboratório, foi maior no presente estudo em comparação ao observado por Pittet e Mendonça. A amostra aqui analisada, no entanto, envolveu a observação de apenas três técnicos e seis médicos, o que impediu comparações muito detalhadas entre as categorias.

Este estudo difere dos demais pelo fato de incluir as mães dos recém-nascidos na avaliação da adesão à lavagem das mãos, pois elas têm papel potencialmente importante na disseminação de micro-organismos por manipularem seus filhos e passarem boa parte do seu dia dentro da UTIN. Deve-se destacar que as mães acompanhantes nesse serviço podem permanecer internadas com os recém-nascidos durante todo o período de sua permanência na UTIN, o que significa que elas são colonizadas com germes hospitalares em poucos dias. No entanto, as mães não participam de manipulações invasivas dos pacientes e, portanto, o risco de elas funcionarem como agentes disseminadores de infecção parece reduzido. Além disso, é preciso 
levar em conta o potencial papel protetor do leite materno na redução do risco de infecções hospitalares, principalmente por seu conteúdo de imunoglobulinas, lisozima, lacoferrina, interleucinas e também pelo fato de que a amamentação promove transmissão de bactérias não patogênicas que podem estimular a resposta imune dos recém-nascidos ${ }^{(18)}$.

No período da manhã, ocorreu maior adesão à lavagem das mãos, resultado similar ao observado em Goiânia ${ }^{(7)}$. Isso pode ser importante no planejamento de intervenções de educação, que provavelmente devem ser mais intensivas com os profissionais que trabalham no período da tarde.

A técnica adequada da lavagem das mãos preconizada pelo manual do Ministério da Saúde ${ }^{(11)}$ foi avaliada de duas formas: por intenção de tratar, que incluiu todas as pessoas observadas, tanto aquelas que lavaram as mãos como aquelas que não as lavaram, sendo estas últimas incluídas na categoria "uso incorreto da técnica", e pelo método de análise por protocolo, que incluiu apenas as pessoas que lavaram as mãos. Comparando-se as duas técnicas de análise, percebe-se que a análise por protocolo ameniza a situação real de certa forma. A análise por intenção de tratar acaba sendo mais fidedigna à realidade encontrada ${ }^{(19-21)}$. Mas, de qualquer forma, nenhum adulto observado lavou a mão com a técnica totalmente correta, o que é bastante preocupante, pois a equipe de enfermagem e os médicos realizam procedimentos invasivos, com grande risco infecções, principalmente em pacientes que apresentam maior suscetibilidade a infecções por micro-organismos já selecionados e que podem ser resistentes a diversas classes de antimicrobianos ${ }^{(15,16,22)}$.

Vários estudos na literatura mundial provam que a melhor forma de prevenir infecções em ambiente hospitalar é a correta lavagem das mãos antes e após a manipulação dos pacientes. Para isso, a melhor maneira de aumentar a adesão dos profissionais de saúde é implantar programas de prevenção de infecção hospitalar ${ }^{(7-17)}$. Com a aplicação de um programa educacional, Lam $^{(14)}$ conseguiu aumentar de 40 para 53\% a adesão de médicos e enfermeiros à lavagem das mãos antes do contato com o paciente e de 39 para $59 \%$ após esse contato. Won ${ }^{(12)}$ conseguiu aumentar a adesão de médicos, enfermeiras e outros profissionais de saúde para $80 \%$ e, com isso, a taxa média de infecções nosocomiais diminuiu de 15,1 por mil pacientes-dia para 10,4 e 11,9 por mil pacientes-dia durante o primeiro e o segundo anos da campanha de prevenção a infecções por meio da lavagem das mãos ${ }^{(12,14)}$.

O presente estudo possui limitações metodológicas que se devem à reduzida amostra observada e à falta de observações noturnas. Além disso, o número de categorias profissionais que estiveram na Unidade durante os períodos de observação foi pequeno, chamando-se a atenção para a não existência de fisioterapeutas, fonoaudiólogas e outros profissionais não pertencentes à Unidade (cirurgião pediátrico, por exemplo). Isso se deve ao fato de a unidade ser pequena e aos períodos relativamente breves de observação. Acredita-se que as outras categorias profissionais, por não pertencerem ao quadro de profissionais que trabalham exclusivamente na Unidade, podem apresentar índices menores de adesão às técnicas de lavagem de mãos. Por outro lado, um dos pontos positivos do presente estudo foi o fato de as observações terem sido realizadas sem o conhecimento prévio dos funcionários, que só assinaram o termo de consentimento livre e esclarecido após o término da coleta dos dados.

Atualmente, o uso de álcool em gel é citado na literatura como uma forma de aumentar a adesão dos profissionais de saúde à limpeza das mãos e diminuir a taxa de infecção hospitalar, pois se gasta menos tempo na higienização das mãos, o produto age mais rápido e é eficaz na erradicação de micro-organismos ${ }^{(9,23)}$. Até o momento da realização do estudo, não se utilizava álcool em gel na Unidade e um resultado concreto do trabalho foi a adoção dessa medida logo após discussão interna, realizada para analisar os dados obtidos.

De qualquer forma, a importância prática do trabalho foi reafirmar a necessidade de se instituirem programas de educação continuada visando a estabelecer e manter a adesão às técnicas e, talvez, campanhas que perpetuem o seu cumprimento. Pode-se concluir, portanto, que as técnicas de lavagem das mãos não são uniformemente seguidas nos hospitais e, por isso, programas educacionais com vistas a aumentar a adesão dos profissionais de saúde à lavagem das mãos são importantes. Mais estudos são necessários para que se avalie a melhor forma de motivá-los.

\section{Agradecimentos}

Os autores agradecem ao Centro Universitário Lusíada (Unilus) pela bolsa de iniciação científica oferecida. 


\section{Referências bibliográficas}

1. Nogueras M, Marinsalta N, Roussell M, Notario R. Importance of hand germ contamination in health-care workers as possible carriers of nosocomial infections. Rev Inst Med Trop Sao Paulo 2001;43:149-52.

2. Dumpis U, Balode A, Vigante D, Narbute I, Valinteliene R, Pirags V et al. Prevalence of nosocomial infections in two Latvian hospitals. Euro Surveill 2003;8:73-8

3. Carvalho ES, Marques SR. Infecção hospitalar em pediatria. J Pediatr (Rio J) 1999;75:S31-45

4. Rojas MA, Efird MM, Lozano JM, Bose CL, Rojas MX, Rondon MA et al. Risk factors for nosocomial infections in selected neonatal intensive care units in Colombia, South America. J Perinatol 2005;25:537-41.

5. World Health Organization. WHO guidelines on hand hygiene in health care. Geneva: WHO; 2006. p. 7-13.

6. Prade SS, Felix J, Mendes A, Gadelha MZ, Pereira M. Estudo brasileiro da magnitude das infecções hospitalares em hospitais terciários. Rev Controle de Infecção Hospitalar 1995;2:11-24.

7. Mendonça AP, Fernandes MS, Azevedo JM, Silveira WC, Souza AC. Lavagem das mãos: adesão dos profissionais de saúde em uma unidade de terapia intensiva neonatal. Acta Sci Health Sci 2003;25:147-53.

8. Pittet D, Mourouga P, Perneger TV. Compliance with handwashing in a teaching hospital. infection control program. Ann Intern Med 1999;130:126-30.

9. Pittet D. Improving adherence to hand hygiene practice: a multidisciplinary approach. Emerg Infect Dis 2001;7:234-40.

10. Stone SP. Hand hygiene: the case for evidence-based education. J R Soc Med. 2001;94:278-81.

11. Brasil. Ministério da Saúde. Normas e manuais técnicos. Lavar as mãos: informações para profissionais de saúde. Brasília: Ministério da Saúde; 1989.

12. Won SP, Chou HC, Hsieh WS, Chen CY, Huang, SM, Tsou KI et al. Handwashing program for the prevention of nosocomial infections in a neonatal intensive care unit. Infect Control Hosp Epidemiol 2004;25:742-6.
13. Pessoa-Silva CL, Hugonnet S, Pfister R, Touveneau S, Dharan S, PosfayBarbe $\mathrm{K}$ et al. Reduction of health care associated infection risk in neonates by successful hand hygiene promotion. Pediatrics 2007;120:e382-90.

14. Lam BC, Lee J, Lau YL. Hand hygiene practices in a neonatal intensive care unit: a multimodal intervention and impact on nosocomial infection. Pediatrics 2004;114:e565-71.

15. Raymond J, Aujard Y. Nosocomial infections in pediatric patients: a European, multicenter prospective study. European Study Group. Infect Control Hosp Epidemiol 2000;21:260-3.

16. Kampf G, Kramer A. Epidemiologic background of hand hygiene and evaluation of the most important agents for scrubs and rubs. Clin Microbiol Rev 2004;17:863-93.

17. Novoa AM, Pi-Sunyer T, Sala M, Molins E, Castells X. Evaluation of hand hygiene adherence in a tertiary hospital. Am J Infect Control 2007;35: 676-83.

18. Silva A, Jones PW, Spencer SA. Does human milk reduce infection rates in preterm infants? A systematic review. Arch Dis Child Fetal Neonatal Ed 2004;89:F509-13.

19. Heritier SR, Gebski VJ, Keech AC. Inclusion of patients in clinical trial analysis: the intention-to-treat principle. Med J Aust 2003;179:438-40.

20. Piaggio G, Elbourne DR, Altman DG, Pocock SJ, Evans SJ, CONSORT Group. Reporting of noninferiority and equivalence randomized trials: an extension of the CONSORT statement. JAMA 2006;295:1152-60.

21. Montori VM, Guyatt GH. Intention-to-treat principle. CMAJ 2001;165: 1339-41.

22. Pessoa-Silva CL, Richtmann R, Calil R, Santos RM, Costa ML, Frota AC et al. Healthcare-associated infections among neonates in Brazil. Infect Control Hosp Epidemiol 2004;25:772-7.

23. Widmer AF. Replace hand washing with use of a waterless alcohol hand rub? Clinical Infectious Diseases 2000;31:136-43. 